\title{
PRIMARY DISCONTINUITIES IN SOLUTIONS FOR DELAY INTEGRO-DIFFERENTIAL EQUATIONS*
}

\author{
HERMANN BRUNNER ${ }^{\dagger}$ AND WENKUI ZHANG ${ }^{\dagger}$
}

\begin{abstract}
In this paper, we prove a number of primary discontinuity results for integral and integro-differential equations with various kinds of delays. Our results are then compared with analogous ones for delay differential equations. Results of this type play an important role in the numerical analysis of functional equations with delay arguments, especially in the design of high-order methods and the analysis of their optimal convergence orders.
\end{abstract}

1. Introduction. Consider the delay differential equation

$$
\begin{aligned}
y^{\prime}(t) & =f(t, y(t), y(\alpha(t, y(t)))), \quad t \in I:=[0, T], \\
y(t) & =\phi(t), \quad t \in[\bar{a}, 0]
\end{aligned}
$$

where $\bar{a}=\inf _{t \geq 0} \alpha(t, y(t)) \leq 0$. Depending on the choice of $\alpha, t-\alpha(t, y(t))$ can be a constant (if $\alpha=t-\tau$ ), a function of $t$ (for example, if $\alpha=q t$ ), or even a function of $t$ and the unknown solution $y(t)$. A remarkable difference between (1.1) and its classical (non-delay) counterpart is that, in general, the solution of (1.1) is determined by an initial function $\phi(t)$ rather than by a simple initial value. As a consequence, even if the functions $f(t, y, x), \alpha(t, y)$ and $\phi(t)$ in (1.1) are $C^{\infty}$-continuous, the solution $y(t)$ is not smoothly linked to the initial function $\phi(t)$ at the point 0 , where in general only $C^{0}$ continuity can be assured. This discontinuity in $y^{\prime}$ is propagated along the integration interval. More precisely, a set of primary discontinuities is generated, whose location is determined by the delayed argument $\alpha(t, y(t))$. This is one of the major challenges when integrating (1.1) numerically as it may cause reduction of accuracy or lead to instability (compare, e.g., [9] and [10]).

In this paper, we will focus on the discontinuity problem for (1.1) with all three kinds of delays, prove discontinuity properties for delay integral and integrodifferential equations, and compare the results with analogous ones for related delay differential equations (1.1). The reader may wish to consult [1]-[3], [5], [7], and [10] and the references listed in these papers (especially [1]) for relevant background material and related results.

2. Discontinuities for delay differential equations. In order to view the discontinuity properties of solutions for delay Volterra equations in the proper perspective, we begin by a brief review of similar results for delay differential equations. Thus, consider the first-order delay differential equation

$$
\begin{aligned}
y^{\prime}(t) & =f(t, y(t), y(t-\tau)), \quad t \in I, \\
y(t) & =\phi(t), \quad t \in[-\tau, 0]
\end{aligned}
$$

where the delay $\tau>0$ is constant. The theory of existence and uniqueness of solutions to (2.1) does not present substantial additional difficulties compared to the ordinary (non-delay) case. This is also true when we consider differential equations with more general delay afterwards, as long as the delay is uniformly strictly positive and does not depend on the solution $y$ itself.

\footnotetext{
*Received November 4, 1997; revised March 9, 1999.

$\dagger$ Department of Mathematics and Statistics, Memorial University of Newfoundland, St. John's, Newfoundland, Canada A1C 5S7 (hermann@math.mun.ca and wenkui@math.mun.ca).
} 
Regarding the solution of (2.1), the most natural method (see also [4]) is called the method of steps (or the method of successive integrations). It consists of first determining the solution $y(t)$ on $[0, \tau]$ from the differential equation without delay,

$$
\begin{aligned}
y^{\prime}(t) & =f(t, y(t), \phi(t-\tau)), \quad t \in[0, \tau], \\
y(0) & =\phi(0),
\end{aligned}
$$

since for $0 \leq t \leq \tau$, the delay argument $t-\tau$ varies in the initial interval $[-\tau, 0]$ and, consequently, the third argument $y(t-\tau)$ of the function $f$ equals the given initial function $\phi(t-\tau)$. Assuming the existence of a solution $y=\phi_{1}(t)$ of this initial-value problem on the whole interval $[0, \tau]$, we proceed recursively by solving

$$
y^{\prime}(t)=f\left(t, y(t), \phi_{n}(t-\tau)\right), \quad t \in[n \tau,(n+1) \tau],
$$

with

$$
y(n \tau)=\phi_{n}(n \tau), \quad n=1, \ldots, M
$$

(we shall assume, without loss of generality, that $T=(M+1) \tau$ for some positive integer $M)$; here, $\phi_{n}(t)$ is the solution of the given initial-value problem on the interval $[(n-1) \tau, n \tau]$.

Thus, questions on the existence and uniqueness of solutions to the delay problem (2.1) reduce to analogous ones for a sequence of initial-value problems for ordinary differential equations. However, smooth data $f$ and $\phi$ in (2.1) will in general not lead to solutions that are smooth on the entire interval of integration.

DEFINITION 2.1. If the solution of (2.1) and its derivatives of order less than, or equal to $\mu$ are continuous at some point $\xi \in I$ but the derivative of order $\mu+1$ is not, then $\xi$ is called a primary discontinuity of problem (2.1).

THEOREM 2.1. The points $\xi_{\mu}:=\mu \tau(\mu=0,1, \ldots)$, are the primary discontinuities of problem (2.1). More precisely, for any sufficiently smooth functions $f$ and $\phi$, $y^{(\mu)}$ is continuous at $\xi_{\mu}$ but $y^{(\mu+1)}$ is, in general, not.

Proof. See [4].

The above results reveal that, as $t$ increases, the solution $y$ of (2.1) becomes smoother. In fact, at the initial point $t=0$, the first derivative $y^{\prime}(t)$ has a primary discontinuity since we have, by $(2.2), y(0)=\phi(0)$ but, in general,

$$
y^{\prime}(0+) \neq \phi^{\prime}(0-) \text {. }
$$

Only for special choices of the initial function $\phi(t)$ is it possible to guarantee continuity of the derivative of the solution at point 0 , for such a function must satisfy the condition

$$
\phi^{\prime}(0-)=f(0, \phi(0), \phi(-\tau)) .
$$

The method of steps can be extended to differential equations with other type of delays, such as multiple delays, variable delay and even state-dependent delay. The difficulty is to locate the primary discontinuities. As a generalization of (2.1), we consider

$$
\begin{aligned}
y^{\prime}(t) & =f(t, y(t), y(t-\tau(t))), \quad t \in I, \\
y(t) & =\phi(t), \quad t \in[\bar{a}, 0]
\end{aligned}
$$


where $t-\tau(t)$ is a strictly increasing function and

$$
0<\tau(t)<t, \quad \bar{a}=\inf _{t \geq 0}(t-\tau(t))
$$

REMARK 2.1. Throughout this paper, when the delay $\tau$ depends on time $t$, we will make this clear by the notation $\tau(t)$. Otherwise, $\tau$ will be a positive constant.

THEOREM 2.2 ([10]). The primary discontinuities of problem (2.4) are generated inductively by the recursion

$$
\xi_{k}-\tau\left(\xi_{k}\right)=\xi_{k-1}, \quad k \geq 1
$$

where $\xi_{0}=0$.

Because of the hypotheses made, a strictly increasing sequence $\left\{\xi_{k}\right\}_{k \geq 0}$ is determined which can in fact be computed a priori by using (2.6). In this way, a sequence of intervals $\left[\xi_{k-1}, \xi_{k}\right]$ is also defined, (see also [10]).

REMARK 2.2. If the functions $\phi(t)$ and $\tau(t)$ in (2.4) have some discontinuities with respect to $t$ in some of their derivatives, then these discontinuities are also propagated by the delay argument $t-\tau(t)$ following the rule (2.6). These discontinuities are called secondary discontinuities.

Further discussion of this topic, especially the case of state-dependent delays, can be found on the following pages; see also [4] for the extension to the multiple delay case.

The existence of primary and secondary discontinuities may lead to a loss of accuracy (reduction of order) or to numerical instability if the mesh underlying a discretization method does not take into account these discontinuities. For a detailed discussion of this problem see, for example, [9] and [10].

3. Discontinuities for delay integro-differential equations. Similar discontinuity results hold for Volterra integro-differential equations with constant delay,

$$
\begin{aligned}
y^{\prime}(t) & =f(t, y(t))+\int_{0}^{t} K(t, s, y(s), y(s-\tau)) d s, \quad t \in I \\
y(t) & =\phi(t), \quad t \in[-\tau, 0] .
\end{aligned}
$$

However, we shall see (compare, for example, Theorem 3.1 and Theorem 2.1) that there are fundamental differences between the regularity of solutions to (3.1) and those of (2.1).

REMARK 3.1. If the delay occurs in one of the limits of integration, for example,

$$
y^{\prime}(t)=f(t, y(t))+\int_{0}^{t} K_{1}(t, s, y(s)) d s+\int_{0}^{t-\tau} K_{2}(t, s, y(s)) d s
$$

or

$$
y^{\prime}(t)=f(t, y(t))+\int_{t-\tau}^{t} K(t, s, y(s)) d s
$$

we can always convert them into the form of (3.1) by a suitable change of variables. So we shall only consider (3.1), without loss of generality. 
THEOREM 3.1. The primary discontinuities of problem (3.1) are the points $\xi_{\mu}:=$ $\mu \tau(\mu=0,1, \cdots)$. To be more precise, the derivative $y^{(2 \mu+1)}(t)$ is discontinuous at the point $\xi_{\mu}$, but lower order derivatives are continuous, provided the given functions functions $f, K$ and $\phi$ are sufficiently smooth.

Proof. The proof is based on the method of steps. In the first interval $[0, \tau]$,

$$
y^{\prime}(t)=f(t, y(t))+\int_{0}^{t} K(t, s, y(s), \phi(s-\tau)) d s .
$$

It is possible to satisfy the condition $y(0)=\phi(0)$, but not, in general, also the condition $y^{\prime}(0+)=\phi^{\prime}(0-)$. The continuity of the derivative of the solution can be guaranteed at the initial point 0 only for deliberately chosen $\phi(t)$, and such a function $\phi(t)$ must satisfy the condition $\phi^{\prime}(0-)=f(0, \phi(0))$.

At the point $t=\tau$, the first derivative of the solution is already continuous. In fact, the derivative

$$
y^{\prime}(t)=f(t, y(t))+\int_{0}^{t} K(t, s, y(s), y(s-\tau)) d s
$$

and the right hand part are continuous functions of $t$ at the point $\tau$, since $y(t)$ is continuous at point 0 . The second derivative,

$$
y^{\prime \prime}(t)=\frac{\partial f}{\partial t}+\frac{\partial f}{\partial y} y^{\prime}(t)+K+\int_{0}^{t} \frac{\partial K}{\partial t}(t, s, y(s), y(s-\tau)) d s
$$

is continuous where we have written $K=K(t, t, y(t), y(t-\tau))$. However, $y^{\prime \prime \prime}(t)$ is not continuous at $\tau$, since it will include $y^{\prime}(t-\tau)$ as a factor, and $y^{\prime}(t-\tau)$ is not continuous at $\tau$ because $y^{\prime}(t)$ is not continuous at 0 .

At the point $t=2 \tau, y^{(4)}(t)$ is continuous, and $y^{(5)}(t)$ is not. At $t=\mu \tau$, we suppose that $y^{(2 \mu+1)}(t)$ is not continuous, and all lower order derivatives are. At $t=(\mu+1) \tau$, differentiate (3.1) $2 \mu+1$ and $2 \mu+2$ times, respectively, to obtain

$$
y^{(2 \mu+2)}(t)=\frac{\partial f}{\partial y} y^{(2 \mu+1)}(t)+\frac{\partial K}{\partial y(t-\tau)} y^{(2 \mu)}(t-\tau)+\text { lower order terms }
$$

and

$$
y^{(2 \mu+3)}(t)=\frac{\partial f}{\partial y} y^{(2 \mu+2)}(t)+\frac{\partial K}{\partial y(t-\tau)} y^{(2 \mu+1)}(t-\tau)+\text { lower order terms. }
$$

According to the hypothesis, $y^{(2 \mu+1)}(t)$ will be continuous at $t=(\mu+1) \tau$, as is $y^{(2 \mu)}(t-\tau)$. As a result, $y^{(2 \mu+2)}(t)$ will be continuous at $t=(\mu+1) \tau$. Unfortunately, $y^{(2 \mu+3)}(t)$ will lose the continuity at $t=(\mu+1) \tau$ as $y^{(2 \mu+1)}(t)$ is not continuous at $t=\mu \tau$. By induction, we know that the derivative $y^{(2 \mu+1)}(t)$ is not continuous at the point $\mu \tau$, but lower order derivatives are continuous under the smoothness assumption for $f$ and $K$.

REMARK 3.2. The difference between Theorem 2.1 and Theorem 3.1 certainly has some numerical implications. When the mesh $\Pi_{N}$ is not constrained, i.e., $h \neq \tau / r$ for some $r \in N$, we can expect a higher convergence order for (3.1) than for (2.1), due to the better regularity properties of the solution for the former problem. 
Consider now

$$
\begin{aligned}
y^{\prime}(t) & =f(t, y(t))+\int_{0}^{t} K(t, s, y(s), y(s-\tau(s))) d s, \quad t \in I, \\
y(t) & =\phi(t), \quad t \in[\bar{a}, 0]
\end{aligned}
$$

where $\bar{a}=\inf _{t \geq 0}(t-\tau(t))<0$. Here, $0<\tau(t)<t$, and $t-\tau(t)$ is strictly increasing. A result similar to Theorem 2.2 holds for (3.6).

THEOREM 3.2. The primary discontinuities of problem (3.6) are generated by the recursion

$$
\xi_{k}-\tau\left(\xi_{k}\right)=\xi_{k-1}, \quad k \geq 1
$$

where $\xi_{0}=0$.

We can also give an analogous result for Volterra integral equations with constant delay of the form,

$$
\begin{aligned}
& y(t)=g(t)+\int_{0}^{t} K(t, s, y(s), y(s-\tau)) d s, \quad t \in I \\
& y(t)=\phi(t), \quad t \in[-\tau, 0]
\end{aligned}
$$

THEOREM 3.3. The primary discontinuities of problem (3.8) are located at the points $\xi_{\mu}:=\mu \tau(\mu=0,1, \ldots)$. More precisely, for any sufficiently smooth data $g, K$, and $\phi, y^{(\mu-1)}$ and lower order derivatives are continuous at $\xi_{\mu}$ but $y^{(\mu)}$ is, in general, not.

Proof. The solution $y(t)$ of (3.8) will in general not be continuous at the initial point $t=0$ : we have continuity if, and only if, $g(0+) \neq \phi(0-)$.

For $t=\tau$, the first derivative is

$$
y^{\prime}(t)=g^{\prime}(t)+K(t, t, y(t), y(t-\tau))+\int_{0}^{t} \frac{\partial K}{\partial t}(t, s, y(s), y(s-\tau)) d s .
$$

Clearly, $y^{\prime}(t)$ is not continuous at $\tau$ whenever $y(t)$ is not continuous at 0 . The remaining argument is similar to that in the proof of Theorem 3.1. We leave the details to the reader.

REMARK 3.3. It is worth noticing that, in contrast to Theorem 2.1, primary discontinuities of the integral equation (3.8) occur in lower order derivatives. lay,

Consider now the neutral Volterra integro-differential equation with constant de-

$$
\begin{aligned}
y^{\prime}(t) & =f(t, y(t))+\int_{0}^{t} K\left(t, s, y(s), y(s-\tau), y^{\prime}(s-\tau)\right) d s, \quad t \in I \\
y(t) & =\phi(t), \quad t \in[-\tau, 0] .
\end{aligned}
$$

THEOREM 3.4. The primary discontinuities of problem (3.9) are given by the points $\xi_{\mu}:=\mu \tau(\mu=0,1, \ldots)$. To be more precise, the derivative $y^{(\mu+1)}(t)$ is discontinuous at the point $\xi_{\mu}$, but lower order derivatives are continuous whenever the functions $f, K$ and $\phi$ are sufficiently smooth. 
The proof of Theorem 3.4 proceeds along the lines of the one for Theorem3.1. We omit the details.

REMARK 3.4. It is known that there is no smoothing to the solutions of neutral delay differential equations (see [4]). However, smoothing indeed happens to the solutions of neutral delay integral and integro-differential equations as is shown in Theorem 3.4.

4. Discontinuities for differential equations with state-dependent delays. Now, consider the state-dependent delay differential equation of the form

$$
\begin{aligned}
y^{\prime}(t) & =f(t, y(t), y(\alpha(t, y(t)))), \quad t \in I, \\
y(t) & =\phi(t) \quad \text { when } \quad t \in[\bar{a}, 0]
\end{aligned}
$$

where $\bar{a}=\min _{t \geq 0} \alpha(t, y(t))$ and $\alpha(t, y(t)) \leq t$ for $t \geq 0$. Classical treatments of (4.1) can be found, for example, in [5] and [8]; compare also [1] and the references listed therein.

The initial-value problem for (4.1) is said to be of continuity class $p \geq 1$, if the following holds over appropriate domains:

1. The mixed partial derivatives $f_{i, j, k}$ are continuous for all $i+j+k \leq p$;

2. The mixed partial derivatives $\alpha_{i, j}$ are continuous for all $i+j \leq p$;

3. $\phi \in C^{p}[\bar{a}, 0]$.

For $l \leq p, C_{l}^{p}[L-\xi, L+\xi]$ is defined by

$$
C_{l}^{p}[L-\xi, L+\xi]=C^{p}[L-\xi, L] \cap C^{p}[L, L+\xi] \cap C^{l}[L-\xi, L+\xi] .
$$

For comparative purposes we recall the following result (and the relevant notation) from (4.1).

THEOREM 4.1. Let the initial-value problem (4.1) have continuity class $p \geq 1$. For $L \in I$, let the integer $l \in[1, p]$ be such that $y \in C_{l-1}^{p}[L-\xi, L+\xi]$ for some $\xi>0$. Assume that there exists a least number $Z \in(L, T)$, such that $Z$ is a zero of integer multiplicity $m \geq 1$ of $\alpha(t, y(t))-L$. Then $y \in C_{q}^{p}[Z-\xi, Z+\xi]$ where $q=p$ if $m$ is even, and $q=\min (p, m l)$ if $m$ is odd.

When the data in (4.1) are in $C^{p}$, we usually expect the solution $y(t)$ to have $p+1$ continuous derivatives except at the primary discontinuities.

The basic idea underlying the proof of Theorem 4.1 is the following. Suppose $\alpha$ is the retarding function and $L \in I$ is a primary discontinuity. We try to find another point $Z L, \alpha(Z, y(Z))-L=0$ and an interval $[Z-\eta, Z+\eta]$, such that the range of $\alpha(t, y(t))$ for $t \in[Z-\eta, Z+\eta]$ covers $[L-\xi, L+\xi]$, a neighborhood of $L$. Then, when we calculate the derivative on both sides of (4.1) and evaluate it at $t=Z$, the discontinuity will appear on the right-hand side because $L=\alpha(Z, y(Z))$ is such a point. As a result, the left-hand side will be discontinuous at $t=Z$ with an order at least one higher. The details may be found in [5].

Before stating the analogous result for Volterra integral equations with variable delays, we briefly look at two special cases contained in Theorem 4.1. The second case shows that not every delay equation leads to primary discontinuities.

1. Constant delay:

$$
\alpha(t, y(t))=t-\tau
$$


where $\tau$ is a positive constant. The discontinuity points are $\mu \tau$ for $\mu=$ $0,1, \ldots$. The solution $y$ satisfies $y \in C_{\mu}^{p}[\mu \tau-\xi, \mu \tau+\xi]$ for some $\xi \in(0, \tau)$. $\mu=l-1, l=\mu+1$. Here, $m=1$ and $m$ is odd. So $q=\min (p, l)=l$.

2. Proportional delay:

$$
\alpha(t, y(t))=q t
$$

with $0<q<1$. It is again independent of the unknown solution $y(t)$. In this case, we cannot find any $t \in[0,+\infty)$ other than zero such that $q t \leq 0$. That is, no primary discontinuities will occur; see also [6]. However, primary discontinuities $d o$ exist if the underlying interval is of the form $I=\left[t_{0}, T\right]$ with $0<t_{0}$. We leave the details to the reader (compare also [1]).

Theorem 4.1 can be extended to Volterra integral equations of the form:

$$
\begin{aligned}
& y(t)=g(t)+\int_{0}^{t} K(t, s, y(\alpha(s, y(s)))) d s, \quad t \in I \\
& y(t)=\phi(t) \quad \text { when } \quad t \in[\bar{a}, 0]
\end{aligned}
$$

where $\bar{a}=\min _{t \geq 0} \alpha(t, y(t))$ and $\alpha(t, y(t)) \leq t$ for $t \geq 0$. Again, by continuity class $p \geq 1$, we mean that the following conditions hold over appropriate domains:

1. The partial derivatives $K_{i, j, k}$ are continuous for all $i+j+k \leq p$;

2. The partial derivatives $\alpha_{i, j}$ are continuous for all $i+j \leq p$;

3. $g \in C^{p}[0, \infty)$ and $\phi \in C^{p}[\bar{a}, 0]$.

Theorem 4.2. Let the data in (4.3) be in $C^{p}, p \geq 1$. For given $L \in I$, let the integer $l \in[1, p]$ be such that $y \in C_{l-1}^{p}[L-\xi, L+\xi]$ for some $\xi$. Assume that there exists a least number $Z \in(L, T)$ such that $Z$ is a zero of integer multiplicity $m \geq 1$ of $\alpha(t, y(t))-L$. Then $y \in C_{q}^{p}[Z-\xi, Z+\xi]$ where $q=p$ if $m$ is even, and $q=\min (p, m l)$ if $m$ is odd.

REMARK 4.1. Note that (4.1) and (4.3) are not identical: differentiation of (4.3) leads to

$$
y^{\prime}(t)=g^{\prime}(t)+\int_{0}^{t} K_{1}^{\prime}(t, s, y(\alpha(s, y(s)))) d s+K(t, t, y(\alpha(t, y(t))))
$$

where $K_{1}^{\prime}=\frac{\partial}{\partial t} K(t, s, y)$.

Proof. [Proof of Theorem 4.2] We proceed along the lines of the proof for Theorem 4.1. Suppose that $L-\xi \leq \alpha(t, y(t)) \leq L+\xi$ for $t \in[Z-\eta, Z+\eta]$. Setting $w(t)=$ $\alpha(t, y(t))$ and $\mathbf{W}(t)=(t, t, y(w(t)))$, then $\mathbf{W}^{(1)}(t)=\left(1,1, y^{(1)}(\alpha) \alpha^{(1)}\right)$, and

$$
\mathbf{W}^{(k)}(t)=\left(0,0, \sum_{Q=1}^{k} v_{k Q} y^{(Q)}(\alpha)\right)
$$

for $k \geq 2$. Here, $\alpha^{(1)}=\frac{d}{d t} \alpha(t, y(t))$ and

$$
v_{k Q}=\sum \frac{k !}{j_{1} ! \cdots j_{k} !}\left(\frac{\alpha^{(1)}(t)}{1 !}\right)^{j_{1}} \cdots\left(\frac{\alpha^{(k)}(t)}{k !}\right)^{j_{k}}
$$


The sum is taken over all $k$-tuples of nonnegative integers $\left(j_{1}, \ldots, j_{k}\right)$ that satisfy $j_{1}+\cdots+j_{k}=Q$ and $j_{1}+2 j_{2}+\cdots+k j_{k}=k$. Denote the scalar function $K_{1}^{(i)}(t, t, y(w(t))$ by $K_{1}^{(i)}$, that is, $K_{1}^{(i)}=K_{1}^{(i)}(\mathbf{W}(t))$. Then

$$
\begin{gathered}
y^{\prime \prime}(t)=g^{\prime \prime}(t)+\int_{0}^{t} K_{1}^{\prime \prime} d s+K^{\prime}+K_{1}^{\prime} \\
y^{(3)}(t)=g^{(3)}(t)+\int_{0}^{t} K_{1}^{\prime \prime \prime} d s+K^{\prime \prime}+K_{1}^{\prime \prime}+K_{1}^{\prime \prime} \\
y^{(k+1)}(t)=g^{(k+1)}(t)+\int_{0}^{t} K_{1}^{(k+1)} d s+K_{1}^{(k)}+\sum_{i=1}^{k} K_{1}^{(i-1)(k-i+1)} \\
(4.6) y^{(k+1)}(t)=g^{(k+1)}(t)+\int_{0}^{t} K_{1}^{(k+1)} d s+K_{1}^{(k)} \\
+\sum_{i=1}^{k} \sum_{q=1}^{k-i+1} \sum \sigma_{k-i+1} \nabla\left(\cdots \nabla\left(\nabla K_{1}^{(i-1)} \circ \mathbf{W}^{\left(i_{1}\right)}\right) \circ \mathbf{W}^{\left(i_{2}\right)} \ldots\right) \circ \mathbf{W}^{\left(i_{q}\right)}
\end{gathered}
$$

Observe that the highest-order derivative of $\mathbf{W}$ occurs when $i=q=1$ in (4.6). (The term is $\left.\nabla K \circ \mathbf{W}^{(k)}\right)$. Consequently the highest order derivative of $y$ in any term on the right-hand side of (4.6) is the $k$-th derivative. Since $y(t)$ is continuous, it follows from (4.5) that $y^{\prime}(t)=y^{(1)}(t)$ is the composition of continuous functions, hence itself continuous, at $t=Z$. Since (4.3) has continuity class $p \geq 1$, it is easy to show by induction from (4.6) that $y^{(k)}$ is continuous at $t=Z$ for all $k \leq l$ since $l \leq p$. (The induction terminates at the $l$-th derivative because $y(\alpha(t, y(t)))$ need not necessarily have more than $l-1$ derivatives at $t=Z$.) This bound on $k$ can be improved.

Let $m$ be even. Then $\alpha(t, y(t))-L$ will remain either nonnegative or nonpositive in some neighborhood of $t=Z$. In other words, $\alpha(t, y(t))$ for $t$ in a neighborhood of $Z$ will not range over intervals containing the jump point $t=L$. Hence $y(\alpha(t, y(t)))$ for $t$ in a neighborhood of $Z$ could have more than $l$ continuous derivatives. It is easy to show by induction from (4.6) that $y^{(p)}$ is continuous at $t=Z$. This establishes the first case.

Let $m$ be odd. Then either $\alpha(t, y(t))-L$ changes sign at $Z$, or $Z$ is a cluster point of zeros of $\alpha(t, y(t))-L$. In either case $Z$ may be a derivative jump point. It is readily verified that the derivatives up to order $m l-1$ of $y(\alpha(t, y(t)))$ that could be discontinuous at $t=Z$ in (4.6) are actually multiplied by appropriate derivatives of order up to $m-1$ of $\alpha(t, y(t))$ which are continuous and which by hypotheses vanish at $t=Z$. Thus the effect of the discontinuities at $t=Z$ is nullified. This completes the proof of Theorem 4.2.

The above proof can be readily modified to establish an analogous result for Volterra integro-differential equations with state-dependent delay,

$$
\begin{aligned}
y^{\prime}(t) & =f(t, y(t))+\int_{0}^{t} K(t, s, y(\alpha(s, y(s)))) d s, \quad t \in I \\
y(t) & =\phi(t) \quad \text { when } \quad t \in[\bar{a}, 0]
\end{aligned}
$$

where $\bar{a}=\min _{t \geq 0} \alpha(t, y(t))$ and $\alpha(t, y(t)) \leq t$ for $t \geq 0$. 
THEOREM 4.3. Let problem (4.7), (4.8) have continuity class $p \geq 1$. For given $L \in I$, let the integer $l \in[1, p]$ be such that $y \in C_{l-1}^{p}[L-\xi, L+\xi]$ for some $\xi>0$. Assume that there exists a least number $Z \in(L, T)$ such that $Z$ is a zero of integer multiplicity $m \geq 1$ of $\alpha(t, y(t))-L$. Then $y \in C_{q}^{p}[Z-\xi, Z+\xi]$ where $q=p$ if $m$ is even, and $q=\min (p, m l)$ if $m$ is odd.

The proof of this theorem is left to the reader.

Acknowledgments. This work was supported in part by the Natural Sciences and Engineering Research Council of Canada (NSERD Research Grant A9406). The authors also gratefully acknowledge the constructive comments of the three anonymous referees on an earlier version of this paper.

\section{REFERENCES}

[1] C. T. H. Baker AND C. A. H. Paul, Pitfalls in parameter estimation for delay differential equations, SIAM J. Sci. Comput., 18 (1997), pp. 305-314.

[2] A. Bellen, One-step collocation for delay differential equations, J. Comput. Appl. Math., 10 (1984), pp. 275-283.

[3] H. BRUNNer, Collocation and continuous implicit Runge-Kutta methods for a class of delay Volterra integral equations, J. Comput. Appl. Math., 53 (1994), pp. 61-72.

[4] L. E. EL'SGOL'TS AND S. B. NoRKIN, Introduction to the Theory and Application of Differential Equations with Deviating Arguments, Academic Press, New York, 1973.

[5] A. FELDSTEIN AND K. W. NEVEs, High order methods for state-dependent delay differential equations with nonsmooth solutions, SIAM J. Numer. Anal., 21 (1984), pp. 844-863.

[6] A. ISERLES, On the generalized pantograph functional-differential equation, Euro. J. Appl. Math., 4 (1993), pp. 1-38.

[7] V. Kolmanovskir And A. Myshkis, Applied Theory of Functional Differential Equations, Kluwer Academic Publishers, Dordrecht, 1992.

[8] K. W. Neves AND A. FELDSTEIN, Characterization of jump discontinuities for state dependent delay differential equations, J. Math. Anal. Appl., 56 (1976), pp. 689-707.

[9] L. ToRelLI, Stability of numerical methods for delay differential equations, J. Comput. Appl. Math., 25 (1989), pp. 15-26.

[10] M. Zennaro, Delay differential equations: theory and numerics, in Numerics of Ordinary and Partial Differential Equations, M. Ainsworth, et al., ed., Clarendon Press, Oxford, 1995, pp. 291-333. 
